\title{
657.
}

\section{NOTE ON THE THEORY OF ELLIPTIC INTEGRALS.}

[From the Mathematische Annalen, t. XII. (1877), pp. 143-146.]

THE equation

$$
\frac{M d y}{\sqrt{1-y^{2} \cdot 1-k^{2} y^{2}}}=\frac{d x}{\sqrt{1-x^{2} \cdot 1-k^{2} x^{2}}}
$$

is integrable algebraically when $M$ is rational: and so long as the modulus is arbitrary, then conversely, in order that the equation may be integrable algebraically, $M$ must be rational. For particular values however of the modulus, the equation is integrable algebraically for values of the form $M$, or (what is the same thing) $\frac{1}{M}$, = a rational quantity \pm square root of a negative rational quantity, say $=\frac{1}{p}(l+m \sqrt{-n})$, where $l, m, n, p$ are integral and $n$ is positive; we may for shortness call this a halfrational numerical value. The theory is considered by Abel in two Memoirs in the Astr. Nach. Nos. 138 \& 147 (1828), being the Memoirs* XIII \& XIV in the Euvres Complètes (Christiania 1839). I here reproduce the investigation in a somewhat altered (and, as it appears to me, improved) form.

Putting the two differentials each $=d u$, we have $x=\operatorname{sn}(u+\alpha), y=\operatorname{sn}\left(\frac{u}{M}+\beta\right)$; and the question is whether there exists an algebraical relation between these functions, or, what is the same thing, an algebraical relation between the functions $x=\mathrm{sn} u$ and $y=\operatorname{sn} \frac{u}{M}$.

Suppose that $A$ and $B$ are independent periods of sn $u$; so that $\operatorname{sn}(u+A)=\operatorname{sn} u$, $\operatorname{sn}(u+B)=\operatorname{sn} u$, and that every other period is $=m A+n B$, where $m$ and $n$ are integers. Then if $u$ has successively the values $u, u+A, u+2 A$, etc., the value of $x$

[* They are the Memoirs xix. and xx. in the Euvres Complètes, t. I., Christiania, 1881.] 
remains always the same, and if $x$ and $y$ are algebraically connected, $y$ can have only a finite number of values: there are consequently integer values $p^{\prime}, p^{\prime \prime}$ for which sn $\frac{1}{M}\left(u+p^{\prime} A\right)=\operatorname{sn} \frac{1}{M}\left(u+p^{\prime \prime} A\right)$ : or writing $u-p^{\prime} A$ for $u$ and putting $p^{\prime \prime}-p^{\prime}=p$, there is an integer value $p$ for which sn $\frac{1}{M}(u+p A)=\operatorname{sn} \frac{1}{M} u$.

Similarly there is an integer value $q$ for which $\operatorname{sn} \frac{1}{M}(u+q B)=\operatorname{sn} \frac{1}{M} u$; and we are at liberty to assume $q=p$; for if the original values are unequal, we have only in the place of each of them to substitute their least common multiple.

We have thus an integer $p$, for which

$$
\begin{aligned}
& \text { sn } \frac{1}{M}(u+p A)=\text { sn } \frac{1}{M} u, \\
& \text { sn } \frac{1}{M}(u+p B)=\operatorname{sn} \frac{1}{M} u .
\end{aligned}
$$

There are consequently integers $m, n, r, s$ such that

$$
\begin{aligned}
& \frac{p A}{M}=m A+n B \\
& \frac{p B}{M}=r A+s B
\end{aligned}
$$

equations which will constitute a single relation $\frac{p}{M}=m$, if $m=s, \quad r=n=0$; but in every other case will be two independent relations. In the case first referred to, the modulus is arbitrary and $M$ is rational.

But excluding this case, the equations give

or, what is the same thing,

$$
B(m A+n B)=A(r A+s B)
$$

$$
r A^{2}-(m-s) A B-n B^{2}=0
$$

an equation which implies that the modulus has some one value out of a set of given values. The ratio $A: B$ of the two periods is of necessity imaginary, and hence the integers $m, n, r, s$ must be such that $(m-s)^{2}+n r$ is negative.

The foregoing equations may be written

$$
\begin{array}{r}
\left(m-\frac{p}{M}\right) A+\quad n B=0 \\
r A+\left(s-\frac{p}{M}\right) B=0
\end{array}
$$

whence eliminating $A$ and $B$ we have

$$
\left(m-\frac{p}{M}\right)\left(s-\frac{p}{M}\right)-n r=0,
$$


that is,

and consequently

$$
\left(\frac{p}{M}\right)^{2}-(m+s) \frac{p}{M}+m s-n r=0
$$

$$
\frac{p}{M}=\frac{1}{2}(m+s) \pm \frac{1}{2} \sqrt{(m-s)^{2}+n r},
$$

where, by what precedes, the integer under the radical sign is negative: and we have thus the above mentioned theorem.

As a very general example, consider the two rational transformations

$$
\begin{aligned}
& z=(x, u, v) ; \text { mod. eq. } Q(u, v)=0 ; \frac{N d z}{\sqrt{1-z^{2} \cdot 1-v^{8} z^{2}}}=\frac{d x}{\sqrt{1-x^{2} \cdot 1-u^{8} x^{2}}}, \\
& y=(z, v, w) ; \text { mod. eq. } P(v, w)=0 ; \frac{M d y}{\sqrt{1-y^{2} \cdot 1-w^{8} y^{2}}}=\frac{d z}{\sqrt{1-z^{2} \cdot 1-v^{8} z^{2}}}:
\end{aligned}
$$

viz. $z$ is taken to be a rational function of $x$, and of the modular fourth roots $u, v$; and $y$ to be a rational function of $z$, and of the modular fourth roots $v, w$; the transformations being (to fix the ideas) of different orders. We have $y$ a rational function of $x$, corresponding to the differential relation

$$
\frac{M N d y}{\sqrt{1-y^{2} \cdot 1-w^{8} y^{2}}}=\frac{d x}{\sqrt{1-x^{2} \cdot 1-u^{8} x^{2}}} \text {. }
$$

Suppose here $w^{8}=u^{8}$, or say $w=\theta u, \theta$ being an eighth root of unity: we then have $Q(u, v)=0, P(v, \theta u)=0$, equations which determine $u$. The differential equation is then

$$
\frac{M N d y}{\sqrt{1-y^{2} \cdot 1-u^{8} y^{2}}}=\frac{d x}{\sqrt{1-x^{2} \cdot 1-u^{8} x^{2}}},
$$

an equation the algebraical integral of which is $y=$ a rational function of $x$ as above: hence, by what precedes, we have

$$
\frac{1}{M N}=\frac{1}{2 p}\left\{m+s \pm \sqrt{(m-s)^{2}+n r}\right\}
$$

a half-rational numerical value, as above.

To explain what the algebraical theorem implied herein is, observe that the equations $Q(u, v)=0, P(v, \theta u)=0$, give for $u$ an algebraical equation. Admitting $\theta$ as an adjoint radical, suppose that an irreducible factor is $\phi(u)$, and take $u$ to be determined by the equation $\phi u=0$; then $v$, and consequently also any rational function $\frac{1}{M N}$ of $u, v$, can be expressed as a rational integral function of $u$, of a degree which is at most equal to the degree of the function $\phi u$ less unity. The theorem is that, in virtue of the equation $\phi u=0$, this rational function of $u$ becomes equal to a halfrational numerical value as above. Thus in a simple case, which actually presented itself, the equation $\phi u=0$ was $u^{2}-4 u+1=0$; and $\frac{1}{M N}$ had the value $u-2$, which in virtue of this equation becomes $= \pm \sqrt{-3}$. 
Thus if the second transformation be the identity $z=y, w=v, M=1$ : we have $v=\theta u$; and the equations are

$$
y=(x, u, \theta u), \quad Q(u, \theta u)=0, \quad \frac{N d y}{\sqrt{1-y^{2} \cdot 1-u^{8} y^{2}}}=\frac{d x}{\sqrt{1-x^{2} \cdot 1-u^{8} x^{2}}} .
$$

In particular, if the relation between $y, x$ be given by the cubic transformation

$$
y=\frac{\frac{v+2 u^{3}}{v} x+\frac{u^{6}}{v^{2}} x^{3}}{1+v u^{2}\left(v+2 u^{3}\right) x^{2}}
$$

so that the modular equation $Q(u, v)=0$ is $u^{4}-v^{4}+2 u v\left(1-u^{2} v^{2}\right)=0$; then, writing herein $v=\theta u$, and taking $\theta$ a prime eighth root of unity, that is, a root of $\theta^{4}+1=0$, we have

$$
Q(u, \theta u)=-2 \theta^{3} u^{2}\left(\theta u^{2}+\theta^{2}+u^{4}\right)
$$

viz. disregarding the factor $u^{2}$, the equation for $u$ is $u^{4}+\theta u^{2}+\theta^{2}=0$; or, if $\omega$ be an imaginary cube root of unity $\left(\omega^{2}+\omega+1=0\right)$, this is $\left(u^{2}-\omega \theta\right)\left(u^{2}-\omega^{2} \theta\right)=0$; so that a value of $u^{2}$ is $u^{2}=-\omega \theta$.

Assuming then $\theta^{4}+1=0, v=\theta u$ and $u^{2}=-\omega \theta$, we have $\left(v+2 u^{3}\right) v=\theta^{3} \omega(1+2 \omega)$, $=\theta^{3} \omega\left(\omega-\omega^{2}\right) ; \quad \frac{v+2 u^{3}}{v}=\omega-\omega^{2} ; \quad \frac{u^{6}}{v^{2}}=\omega^{2}, \quad\left(v+2 u^{3}\right) v u^{2}=-\omega^{2}\left(\omega-\omega^{2}\right), u^{8}=\omega^{4} \theta^{4}=-\omega ;$ and the formula becomes

giving

$$
y=\frac{\left(\omega-\omega^{2}\right) x+\omega^{2} x^{3}}{1-\omega^{2}\left(\omega-\omega^{2}\right) x^{2}}
$$

$$
\frac{d y}{\sqrt{1-y^{2} \cdot 1+\omega y^{2}}}=\frac{\left(\omega-\omega^{2}\right) d x}{\sqrt{1-x^{2} \cdot 1+\omega x^{2}}}
$$

where as before $\omega^{2}+\omega+1=0$, a result which can be at once verified. We have $\left(\omega-\omega^{2}\right)^{2}=-3$; or the coefficient $\omega-\omega^{2}$ in the differential equation is $=\sqrt{-3}$, which is of the form mentioned in the general theorem.

We might, instead of $z=y$, have assumed between $y$ and $z$ the relation corresponding to any other of the six linear transformations of an elliptic integral, and thus have obtained in each case, for a properly determined value of the modulus, a cubic transformation to the same modulus.

Cambridge, 10 April, 1877. 